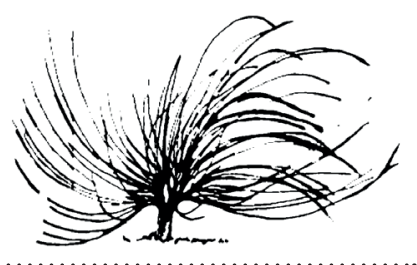

\title{
¿Hay espacio para el uso de animales en la enseñanza de la biología? Reflexiones desde una perspectiva ecológica y multinivel
}

\author{
Bianca Camila De Giuseppe \\ Universidad Nacional de San Luis \\ Argentina \\ camidegiuseppe@gmail.com \\ Martín Gonzalo Zapico ${ }^{2}$ \\ Instituto de Formación Docente Continua \\ Universidad Nacional de San Luis \\ Argentina \\ athenspierre@gmail.com
}

\begin{abstract}
Resumen
En este ensayo abordaremos las problemáticas vinculadas a la manipulación de animales para la enseñanza de la biología. Partiendo de un modelo ecológico, se sostiene la hipótesis de que el empleo de animales para la enseñanza de la biología no solo no es ético, sino que tampoco es rentable, eficaz, ni favorece el desarrollo de habilidades
\end{abstract}

\section{$(0 \Theta \Theta$}

Recibido: 15 de abril de 2019. Aprobado: 28 de octubre de 2019.

http://dx.doi.org/10.15359/rep.15-1.1

1 Es alumna avanzada de la carrera de Licenciatura en Ciencias Biológicas de la Universidad Nacional de San Luis. Se desempeña como pasante en investigación y docencia. Ha asistido a numerosas reuniones científicas.

2 Es profesor en Letras de la Universidad Nacional de Mar del Plata. Se desempeña como profesor responsable en el Instituto de Formación Docente Continua y como becario doctoral en la Universidad Nacional de San Luis. Ha publicado dos libros, numerosos artículos en revistas científicas, y ha asistido a numerosas reuniones científicas. ORCID: https://orcid. org/0000-0002-0604-9943 
sociales fundamentales como la empatía, llegando, incluso, a manifestarse efectos psicológicos adversos en estudiantes expuestos a dichas situaciones. Para la apoyatura de este enunciado, se acudirá a evidencia teórica, práctica y experimental. Concluimos, a partir de nuestro análisis, que la eliminación de las prácticas que involucran la manipulación de animales favorecería no solo a los mismos animales y a quienes las ponen en práctica, sino a la sociedad en su conjunto, pues se estarían formando, desde los primeros años de escolarización, sujetos con inteligencia ecológica y empatía animal, rasgos que favorecen un trato amable y ético hacia el medio ambiente.

Palabras clave: pedagogía, biología, ecología, manipulación de animales, ética.

\begin{abstract}
In this essay, we will approach the problems related to the manipulation of animals for the teaching of biology. Based on an ecological model, a hypothesis is held that the use of animals for the teaching of biology is not only unethical, but also not profitable, effective, and it does not favor the development of fundamental social skills, such as empathy, even reaching such an extent that students exposed to these situations can manifest adverse psychological effects. To support this statement, we will go to theoretical, practical, and experimental evidence. We conclude, from our analysis, that the elimination of practices that involve the manipulation of animals would favor not only the animals themselves and the students, but society as a whole, since they would be teaching from the first years of schooling, students with ecological intelligence and animal empathy, traits that favor a friendly and ethical treatment towards the environment.
\end{abstract}

Keywords: pedagogy, biology, ecology, animal handling, ethics. 


\section{Introducción}

$\mathrm{E}$ 1 estudio de las ciencias naturales como parte de una currícula de nivel primario y secundario es un fenómeno que se registra en los niveles mundial, regional y local (Carin y Sund, 1993; Nosofky y McDaniel, 2019), donde los principales debates están puestos en la epistemología y los enfoques empleados para el dictado de dicha materia o conjunto de materias.

Así, se encuentran polémicas sobre el lugar de la teoría de la evolución (Rutlledge y Mitchell, 2002) que abarcan posturas desde la necesaria obligatoriedad de un enfoque netamente científico y laico de las ciencias naturales como cimiento para una forma crítica y amplia de entender la ciencia en su totalidad (Sinatra, Brem y Evans, 2008), hasta posiciones más moderadas, las cuales promueven los matices teóricos en el contexto de una enseñanza que siempre estará vinculada directamente con los valores sociales del medio (Anderson, 2007), tal como sucede en instituciones de enseñanza con perspectiva religiosa. También, se hallan fuertes discusiones alrededor del papel de la biología en relación con las denominadas teorías con perspectiva de género (García, 1994; Vázquez-Cupeiro, 2015), donde se observan posturas que fluctúan entre una necesaria ausencia total de la perspectiva de género en la enseñanza de la biología (Corredor, 2019; Eagly, 2018), hasta las que buscan hacer obligatoria, incluso para contextos religiosos, la perspectiva de género en la enseñanza de las ciencias naturales (Lane, 2016; Szyrom, 2017), fundamentándose en que es la única forma de acceder a una noción realmente igualitaria de la enseñanza de las ciencias naturales. Todos estos posicionamientos podrían confundir, parcializar e impedir el derecho de la niñez y la juventud a información científica y sistemática.

Por otra parte, hace décadas (Paterson, 2017) se viene disputando un encarnizado debate alrededor del concepto de "calentamiento global", el cual condensa posturas totalmente catastróficas que señalan un casi inevitable aniquilamiento de la sociedad humana en los próximos decenios (Herndon, 2015), al punto de hablar de algo ya irreversible. Se pasa por opiniones que, con un enorme arraigo crítico, buscan entender y prevenir a la sociedad por los cambios negativos (aunque no catastróficos) que se verán en próximos años, a raíz de este fenómeno (Coss, Salvador y Salazar, 2017). Igualmente, están aquellos que se colocan en 
una postura negacionista respecto a dicho fenómeno, quienes, si bien no tienen representantes fuertes en el nivel académico, sí cuentan con una gran presencia en redes sociales, portales, noticias de Internet y demás sitios que, como ya ha sido ampliamente estudiado (De Pablo, 2016; Flichtentrei, 2017; Candon-Mena, 2018), moldean la opinión pública aún más que la comunidad científica y sus hallazgos, así como llegan, incluso, al campo de la ecología y su enseñanza.

Ahora, se conviene que, al discutirse este asunto, no se habla solo de la validez o no del término para referir a un proceso negativo de cambio de temperatura en la superficie terrestre, sino que se extiende también a la pregunta ¿cuál es el lugar del ser humano en relación con el medio ambiente?, y a la interrogante pedagógica ¿cómo se enseña a las futuras generaciones a tratar al medio ambiente? La educación con perspectiva ecológica, llamada a veces educación ambiental (Novo, 1996; Sauvé, 1999; Salgado, 2007), abarca toda una serie de prácticas que refieren no solo a una formación consciente y cuidadosa respecto del medio ambiente, sino también a una con perspectiva de desarrollar economías y sociedades sustentables (Villaverde, 2009), las herramientas de enseñanza-aprendizaje vinculadas al uso y gestión de la tierra (Rodríguez Marín et al., 2017), la bioética y el debate por el estatuto ontológico de los animales (Bustos y Valenzuela, 2018), entre muchos otros asuntos.

Asimismo, es en el marco de esta perspectiva que se propone debatir el uso de animales para la enseñanza de las ciencias naturales en los niveles primario y secundario del sistema educativo. Se cree que una visión ecológica de la enseñanza de las ciencias naturales no solo es necesaria, sino también útil en términos económicos, eficaces y psicológicos. Además, se valora, así como sostienen William Magnusson et al. (2013), que es necesaria una panorámica integradora de todos los actores involucrados en los procesos que buscan vincular estudios referentes al medio ambiente con sus beneficiarios tanto directos como indirectos, en este caso, la sociedad en su conjunto. Esta perspectiva amplia y fundamentada en hechos concretos (Zapico, 2019) permite hipotetizar beneficios tanto para el individuo como para su entorno familar, docentes, la institución educativa y otros beneficiarios en distintas escalas de análisis. Es decir, lo ético no estaría dado por la ausencia total del empleo de animales para la enseñanza, sino por la 
reflexión en torno a cómo ese uso es justificable, en la medida en que no hay realmente otra alternativa más económica o eficaz.

Para este propósito, se realizará un recorrido que comenzará con la conceptualización del uso de animales en la enseñanza de la biología, mediante la exposición de distintas posturas y abordajes epistemológicos que la sustentan. Luego, se expondrán argumentos sobre aspectos eficaces, económicos y psicológicos vinculados a dicha práctica. Finalmente, se presentarán los beneficios derivados de una alternativa más ecológica y que tienda no a la desaparición de animales en la enseñanza de la biología, sino a otros tipos de uso.

\section{El uso de animales para enseñar biología}

¿A qué se refiere la práctica de usar animales en la enseñanza? Antes de poder situar cuál es exactamente el lugar en el que este ensayo se enmarca, es pertinente definir aquellos sitios de los cuales se aleja la perspectiva defendida en este escrito.

Peter Singer (2018), fundador y defensor del movimiento "Liberación Animal", sostiene que una postura realmente ética para con los animales implicaría una homologación total entre los derechos humanos y los animales. En su propia perspectiva, el uso de animales para la enseñanza en cualquier nivel educativo está descartado, pues los conceptos de "libre de crueldad" (Bustos y Valenzuela, 2018) o "uso ético" (de Martínez y de Osorio, 2015) no son más que eufemismos que enmascaran una diferenciación de hecho y una actitud tanto paternalista como jerarquizante.

Por otra parte, se ubican los enfoques que ven absolutamente necesario el trabajo con animales vivos en disciplinas como veterinaria, medicina y biología (Maldonado-Villamizar y Aquino-Guerra, 2016), los cuales a su vez extienden dichas premisas a los niveles de formación primario y secundario, pues aseguran que el trato con animales es fundamental para el desarrollo de la empatía; incluso, en la manipulación que deriva en muerte, se está aportando a una educación con perspectiva ecológica. Este tipo de argumentación, además, suele verse reforzada por una inercia en la forma de plantear los procesos de enseñanza-aprendizaje. Esta postura se ve respaldada, como bien señala Ortiz (2016), con la justificación pedagógica fuertemente vinculada a la tradición. 
Es así como hay docentes que justifican sus prácticas basadas en cómo las impartieron sus maestros $\mathrm{y}$, al considerarse a sí mismos bien formados, evalúan que sus estudiantes deben aprender de la misma forma, incluso cuando hay evidencia de que dicho método no es el óptimo (Viñao, 2000), pues los alumnos tienden a encontrar afinidad en los métodos de aprendizaje a los que se les ha expuesto; estos se repiten y se perpetúan. A partir de esto, la tradición sigue justificando la práctica. Sin embargo, el hecho de que una práctica sea tradicional no justifica su continuación ni garantiza su efectividad (Pérez, 2010). La utilización de este argumento en la educación, de manera general, se podría suponer, promueve el conservadurismo y la fosilización pedagógica, lo que obstaculiza el desarrollo y la promoción de nuevas técnicas de enseñanza-aprendizaje posiblemente más efectivas.

Ahora bien, ¿esto implica necesariamente que todos los usos de animales son reprobables o imperativos? Para la definición de lo que se considera un uso realmente ético y humano de animales, que a veces resulta útil o necesario, se toma la distinción metodológica realizada por Von Arcken (2017), al referirse a la formación escolar y universitaria en el empleo de animales. Como primer punto, una separación entre lo puramente ético y lo metodológico, en la que ambas partes deben coincidir para llevar a cabo la utilización de un animal. Es decir, el planteo ético del ser sintiente no basta para enfocar o definir el uso de animales (pues si así fuera, simplemente no se usarían y ya), sino que debe considerarse la dimensión metodológica de dicha ética. La pregunta que debe hacerse es ¿no hay, metodológicamente hablando, otra forma de enseñar eso que quiero? Si la respuesta es no, corresponde cuestionarse: jes ético el trato que estoy dando al animal en esta práctica? En esta perspectiva, la formación y el aprendizaje como fines no justifican todos los medios, sino que solo aquellos en los cuales el énfasis está puesto en el bienestar y trato digno del animal.

Un segundo punto de esta perspectiva se relaciona con evidenciar el mito ampliamente extendido de que por saber realizar experimentos, pruebas, intervenciones o manipulación de animales se los sabe tratar. Llegar a conocer procedimientos y protocolos para el empleo de animales no asegura la capacidad del docente o investigador de dar un trato ético y digno a estos. Como se ha señalado en diversos estudios (Campos, Pasquali y Peinado, 2008; Rigo y Donolo, 2013; Calderón-Amor, 2015; Galaz, Ayala y Manrique, 2017), variables como la 
empatía animal o la inteligencia natural no están involucradas con un conocimiento científico-técnico, sino más bien con rasgos inherentes de la persona, como una formación escolar activamente ecológica o con conciencia medioambiental, o el contacto temprano y constante con distintos tipos de animales, sean domésticos o salvajes. Es decir, no se debe dar por hecho que la formación de grado o posgrado del profesora lo ha capacitado en ética y trato digno. La formación en ética y bioética, como señalan Garzón y Zárate (2015), tiene un lugar minoritario o a veces inexistente en los planes de estudio de distintas carreras, incluidos los profesorados. Dada esta situación, lo correcto sería procurar dichos tipos de capacitaciones, fundamentándose en protocolos internacionales ampliamente aceptados.

Algunos de dichos protocolos vigentes en la Unión Europea y Estados Unidos, que pueden consultarse en el documento de la Academia Nacional de Medicina de México (1999), traducido del de Washington, son a) Guía para el cuidado y uso de animales de laboratorio, auspiciada por el National Institute of Health (NIH), USA, en sus diferentes versiones (la última edición fue publicada por The National Academy Press, EE. UU., en el 2011); b) Directiva 86/609/CEE relativa a la aproximación de las disposiciones legales, reglamentarias y administrativas de los Estados Miembros de la Comunidad Económica Europea respecto a la protección de los animales utilizados para experimentación y otros fines científicos; c) Convención ETS 123 para la protección de animales vertebrados usados para experimentación y otros propósitos científicos, adoptada por el Consejo de Europa, el cual tiene fuerza de recomendación, y d) en el nivel internacional existen recomendaciones sobre categorización, educación y entrenamiento mínimo requerido por todas las personas relacionadas con el cuidado y uso de animales de laboratorio, por ejemplo, las elaboradas por la Federación Europea de Asociaciones de Ciencia de Animales de Laboratorio (FELASA).

Lo anterior quiere decir que la incorporación de normativas de índole general al terreno de lo pedagógico y didáctico permitirá ampliar no solo las bases éticas del trato hacia los animales en múltiples situaciones, sino informar a los involucrados sobre las problemáticas que se están ocultando o dejando de lado, al no hablar de estos asuntos. Si bien la educación ecológica es el eje de muchos planes de estudios y propuestas de formación en los niveles primario, secundario y 
universitario, las prácticas observadas reflejan un desconocimiento o desinterés por ahondar en las problemáticas.

Un tercer punto consiste en el inconveniente que representa, incluso en las mejores condiciones y con el trato óptimo, el empleo simultáneo de un mismo espécimen para distintos tipos de procedimientos. Muchas veces, y principalmente por razones de optimización de costos, se busca emplear la menor cantidad de animales posible para la mayor cantidad de procesos. En contraposición a este comportamiento (que tiende a registrarse más en ámbitos de escuela secundaria o primaria, donde la regulación es menor), se propone la incorporación de alternativas más eficaces, baratas y éticas para el trato de los animales. Es decir, sin llegar a un punto de reemplazo total, se da alternativas que suplan la falta de disponibilidad, tales como el empleo de laboratorios web o situaciones de uso no invasivo de animales.

Ya se han señalado los ejes sobre los cuales se cimenta nuestra postura ética de la utilización de animales. Como se mencionó, a estos lineamientos le sumaremos el enfoque multinivel propuesto por Magnusson et al. (2013), que señala la necesidad de generar información y formación en temas relativos a biodiversidad y conciencia ambiental que lleguen a actores en distintos niveles del sistema social; y la propuesta tanto ética como estadística de Zapico (2019), la cual, en el marco de un programa democrático, plantea la investigación sistemática y revisión de evidencia empírica para la superación de los particularismos en temáticas que atañen a la sociedad en su conjunto.

\section{El problema económico}

Las dimensiones económicas vinculadas al empleo de animales para la enseñanza son tan grandes que, para comprender la gravedad de los costos asociados, es necesario segmentarla en, al menos, dos momentos distintos: la adquisición de los animales y su posterior manutención y alojamiento. Hay, primordialmente, dos vías conducentes a la adquisición de animales para usos de enseñanza: una, mediante la construcción y el sostenimiento de un bioterio o dos, a través de la compra de dichos animales a bioterios o criaderos especializados. En ambos casos, una vez obtenidos los animales, deben ponderarse los costos vinculados a la infraestructura necesaria para su alojo, logística y manutención. 
Para dimensionar los costos asociados a la constitución y mantenimiento de un bioterio, tomaremos como referencia el presupuesto manejado por la Universidad Nacional de Quilmes (provincia de Buenos Aires, Argentina) en el 2017, construido de acuerdo con lo estipulado en el Proyecto de Ley S-0048/18, que a su vez se adscribe a los principios éticos del uso de animales establecidos en la Ley 14.346 (Malos Tratos y Actos de Crueldad a los Animales). Esta última estipula solo para la construcción (sin contar la adquisición de un terreno o espacio) al menos 500000 dólares de inversión que luego se ve cargada por unos 150000 dólares, con miras a la manutención anual del bioterio. Si bien es cierto que un bioterio funcional puede generar una serie de ingresos a través de la cría y venta de animales para diversos usos, los costos generados a partir del personal encargado de la crianza y cuidado de los especímenes son bastante elevados; además, a menos que el proyecto se sustente con inversiones estatales (Argentina) o de empresas privadas interesadas (Chile), difícilmente será viable y mucho menos aún para una institución escolar del nivel medio o inicial.

Esto conlleva pensar la vía de la adquisición de animales para su uso, a través de la compra a un bioterio o un criadero, y luego hacerse cargo del coste de mantenimiento para el alojamiento de los especímenes. De acuerdo con un documento presupuestario específico publicado por Castillo et al. (2005), la adquisición de unos 100 especímenes junto con las condiciones necesarias para su alojamiento durante 180 días insumen un costo aproximado de 8000 dólares.

Una alternativa más económica podría consistir en la compra de los animales necesarios y tercerizar el monto del alojamiento y mantenimiento a un bioterio o centro de instrumentación científica (como el de la Universidad de Granada). El costo de mantenimiento mínimo de un especímen (puede ser rata, cobaya, rana, aquí se ha realizado un promedio) por día es de 1,20 dólares (unos 216 dólares por los 180 días), a lo que se suma la adquisición de los especímenes a razón de 2 dólares por unidad (unos 200 dólares). Los precios combinados dan un total de 416 dólares, lo cual es significativamente bajo, si lo comparamos con las opciones anteriores. No obstante, a esto debe sumarse la logística y todo el equipamiento e infraestructura necesarios para el trabajo con esos animales, que implica una partida presupuestaria aparte. Esto sin contar la posibilidad de acceso al tipo de establecimientos que proveen tanto especímenes como servicios de alojamiento. Si partimos 
de la idea de buscar un procedimiento estandarizado y económico de enseñanza de la biología, los circuitos vinculados con la adquisición de animales no parecieran ser los apropiados.

Ahora bien, estos números poco significarían, si no se les compara con otras elecciones posibles para la enseñanza y formación no basadas en animales. Como métodos alternativos existentes (y complementarios entre sí), podemos nombrar: incorporación de Internet orientada a la búsqueda y al empleo de información multimedia (fotos, videos); simulación computacional 3D, mediante plataformas de laboratorios virtuales (fundamentalmente, realidad aumentada).

El primer grupo de métodos, que ya ha sido ampliamente documentado (Álvarez y Carlino, 2004; Llach, Del Mar y Aris, 2005; Rodríguez et al. 2012), no se propone como un reemplazo total de la práctica de laboratorio, sino que atañe exclusivamente a los experimentos que emplean animales para su uso. Las propuestas centrales en este tipo de programas giran en torno al concepto de los métodos de aprendizaje por resolución de problemas tendientes al desarrollo de la autonomía en los procesos de enseñanza-aprendizaje. Puntualmente, el estudio de Llach, Del Mar y Aris (2005) citado describe el proceso de incorporación del Internet al laboratorio y al aula, donde la búsqueda de material en video no solo amplía el concepto, ya bastante arcaico, de bibliografía de trabajo en la clase, sino que permite dar matices de autonomía al estudio de las ciencias biológicas, pues la búsqueda de herramientas de consulta de distinto tipo (guiada y sistemática) deja espacio para los caminos personales, las inquietudes, preguntas nuevas, generación de trabajo en grupo de forma espontánea, circulación de la información entre pares, entre muchos otros beneficios. Sobre la presencia de Internet en la escuela, así como los costos asociados, hay una enorme cantidad de material escrito (Adell, 1996; Gutiérrez y Bacallao, 2003; Momiló, Siganés y Meneses, 2008; Martínez, 2015; Monteiro, 2015; Rocavado, Otavianelli y Cadena, 2017, entre muchísimos otros) del cual se extraen las siguientes conclusiones, en términos tanto de implementación como de costos. Primero, respecto a la posibilidad de acceso al recurso, tanto en Argentina como en América Latina la tendencia al empleo del multimedia con ingreso a Internet es creciente, y ya está estipulada en los lineamientos nacionales de muchos ministerios de educación la presencia en todos los establecimientos educativos de conexión a Internet y ordenadores para su empleo. Segundo, los costos 
vinculados al pago y sostenimiento del servicio (junto con los costos de mantenimiento) son prácticamente nulos y absorbidos directamente por el presupuesto de las instituciones, sin representar riesgos económicos. Además, incluso si los costos fueran más altos, hay políticas y programas estatales, en el nivel América Latina, que facilitan y subsidian el acceso a este recurso. Finalmente, el funcionamiento de las instituciones educativas en general ya presupone el empleo de Internet, pues la tendencia a la digitalización y almacenamiento remoto de la información ha permitido tanto la optimización del espacio como la disminución en la producción innecesaria de papel. En pocas palabras, los precios derivados de la incorporación de tales instrumentos tienden a cero.

Ahora bien, hoy día el acceso a Internet no es la única posibilidad para cambiar la perspectiva en la enseñanza de la biología. Puntualmente, y con miras a reemplazar el empleo de animales de laboratorio, distintas empresas han desarrollado toda una serie de herramientas didácticas, pero que pueden enmarcarse en el concepto de "laboratorio virtual", el cual dispone de tecnología de realidad aumentada. Los altos costos en la instalación y el mantenimiento de laboratorios en instituciones escolares (Vázquez, 2009; Masco Jové, 2013; Ruiz y Liseth, 2019) han llevado a la progresiva búsqueda de alternativas más económicas, entre las cuales los que más se han destacado por su efectividad y viabilidad (Gil y Di Laccio, 2017) han sido los laboratorios virtuales. Estos, a su vez, pueden dividirse en dos grandes tipos según su interfaz, soporte y posibilidades: 1 . aquellos que son aplicaciones y pueden instalarse y emplearse en un teléfono inteligente de gama media; 2. los que constituyen verdaderas plataformas virtuales de aprendizaje, con tecnología de realidad aumentada y que permiten llevar a cabo simulaciones en tres dimensiones en tiempo real (Calderón et al., 2015). Contra todo prejuicio y diagnóstico, estudios que buscaron evaluar no solo la incorporación, sino también el nivel del aprendizaje en estos medios nuevos, como el llevado a cabo por Noboa y Brito (2016), han puesto de manifiesto el carácter evidentemente más económico y las mejoras en términos de rendimiento académico cuando se evalúan prácticas vinculadas a conocimientos propios del laboratorio. Cabe destacar que, entre todas las funciones y posibilidades que estos laboratorios virtuales poseen, se encuentran además simulaciones de experimentos con fauna que reemplazan en su totalidad la necesidad de adquisición y 
almacenamiento de animales, procesos que muchas veces no se realizan en las condiciones óptimas y producen daños a los especímenes.

Esta última observación lleva directamente al segundo gran argumento que aquí se propone a la hora de reducir al mínimo el uso de animales de laboratorio para la enseñanza de la biología, el problema de la eficacia. ¿Es realmente más efectivo el método tradicional? Es importante hacer un análisis para conocer lo que muestran los estudios comparativos.

\section{El problema de la eficacia}

Dado que no hay estudios específicos en los cuales se contrasten dos grupos a efectos de la resolución de determinados problemas vinculados con el aprendizaje de la biología (solo uno aprendiendo en un laboratorio real con manipulación de animales reales y otro, mediante laboratorios virtuales de alta complejidad), para comparar, en alguna medida, la eficacia de uno u otro modelo, se debe acudir a evidencia indirecta o teórica, en este caso, de contraste.

En el ya señalado estudio de Noboa y Brito (2016), se midió el desempeño de dos grupos de adolescentes en una prueba enlazada a contenido de clase impartido en el laboratorio. En un caso, la enseñanza estuvo dada de forma tradicional; en el otro, se efectuó un modelo de participación vía laboratorio virtual. El segundo grupo obtuvo puntuaciones mejores, incluso en términos de significancia estadística. Si bien puede aducirse que se trata de un $\mathrm{N}=50$ bajo y poco representativo, se condice con lo analizado por Paredes (2018), quien estudió la mejora en puntuaciones vinculadas a ciencias, a través de la implementación del proyecto STEM (Science, Technology, Engineering and Mathematics) y el aprendizaje basado en proyectos promotores de la tecnología que reemplazaría las formas tradicionales de enseñanza.

Otro ejemplo reciente y destacado es el del equipo de $\mathrm{Mu}-$ ñoz-López (2018), quien desarrolló, para la enseñanza primaria y secundaria, un modelo de laboratorio mínimo y económico, desprovisto de animales, complementado con el uso de software específico de laboratorio. Su proyecto, denominado "Laboratorio Nómada del Agua", ha representado una verdadera revolución en términos de democratizar el acceso a la ciencia, pues ha demostrado que con una cantidad relativamente limitada de recursos se puede dar formación de calidad en ciencias, así como promover el interés por el medio ambiente y 
la conciencia ecológica. Mediante un análisis de citas a través de un método de coocurrencia, a través del software Atlas.Ti, se demostró el desarrollo de una conciencia ecológica, interés por el cuidado del agua y empatía por el medio ambiente, aspectos increíblemente altos, en especial cuando se los comparó con textos previos a la intervención (incluso en escuelas y con alumnos que disponían de equipamiento).

Un asunto pocas veces indagado, y que también habla de una eficacia comparada muy superior en favor del empleo de laboratorios virtuales o modelos mixtos, es el de las posibilidades de simulación. En un laboratorio tradicional, los procedimientos, desafíos y objetivos tienden a ser los mismos, y, en el caso de los niveles primario y secundario, se ven reducidos a algunos experimentos rutinarios. Maricela et al. (2018) ampliaron las posibilidades y limitaciones clásicas, al incorporar el trabajo con granjas virtuales que posteriormente se convertirían en una experiencia real, donde no solo se desarrollaron las competencias esperadas por las autoridades nacionales, sino que también se generó (incluso por arriba de las expectativas) un interés muy grande en la niñez por la biología y la química. En la parte de análisis curricular de su estudio, dejaron en claro que los conocimientos, competencias y herramientas que se esperaba los estudiantes desarrollen a través de su paso por el nivel primario veían un desarrollo acelerado, cuando se implementaba este tipo de experiencias. La diferencia no estaba dada por la presencia o no del laboratorio en las escuelas, sino por la incorporación de esa dimensión virtual (actualmente muy próxima a los jóvenes) al contexto de enseñanza-aprendizaje.

Un ejemplo puntual del uso de animales como un método innecesario es el estudio realizado por Filkenstein et al. (2005), en el cual se analizó el efecto de sustituir el empleo de un laboratorio clásico a la hora de realizar tareas como disección, contra la utilización de modelos computarizados. Para ello, compararon el desempeño de dos grupos en pruebas tanto de conocimientos teóricos como de habilidades prácticas y observaron que los integrantes del grupo entrenado mediante métodos de simulación tuvieron un desempeño abrumadoramente superior a su contraparte entrenada con formas tradicionales.

Más allá de los innumerables ejemplos que podrían continuarse citando, queda bastante claro cómo la incorporación de las alternativas virtuales no solo resulta, en primera instancia, más barata, sino también más eficaz. Tales opciones permiten la concreción del acceso al 
conocimiento o las competencias que se espera desarrolle la juventud, así como el fomento del interés por la ciencia y la ecología. A continuación, se hablará del aspecto psicológico y de por qué del uso de animales en la enseñanza de la biología debería ser limitado a lo estrictamente necesario.

\section{El problema psicológico}

Desde una perspectiva del bienestar psicológico (Millán, 2016), hay evidencia de que el uso de animales en educación puede tener efectos adversos sobre el estudiantado; se muestra que forzarlo a participar en dichas prácticas de laboratorio puede tener efectos psicológicos nocivos, aspectos para los cuales los docentes no suelen preparar a los educandos, ni aun ellos mismos están capacitados para atender.

Capaldo (2004), en una revisión del tema, cita una gran cantidad de estudios con ejemplos concretos en los cuales se observa de forma directa, y en algunos casos con consecuencias psicológicas graves, los efectos nocivos de la manipulación forzada de animales. Entre los puntos que ella destaca el fundamental es la tendencia natural de los niños a no querer dañar animales (incluso a cortar o intervenir animales ya muertos), mostrada en llantos, vómitos, manifestaciones de violencia o la simple y llana negación a la tarea. Otro punto muy interesante señalado es el tema de las creencias. Un infante de seis años ya es capaz de discernir, en buena medida, qué está bien o mal (al menos en su marco de referencia), y el forzar a niños con convicciones por arriba de la media respecto al valor de los animales a someterlos a tareas estresantes, dañinas o incluso letales puede afectar sobremanera su autoestima, a veces de forma irreversible. Un tercer punto (entre otros que se recomienda leer en el artículo) es el desarrollo observado, en algunos alumnos que se vieron forzados a dañar a perros contra su voluntad, de una apatía aprendida hacia los animales, en general, y hacia los domésticos, en particular. Este tipo de respuesta (la pérdida de empatía con los animales) no puede pasar desapercibida y mucho menos en un nivel inicial e incluso secundario de educación. Tanto en el DSM (Manual de Diagnóstico y Estadística para enfermedades mentales, por su traducción al español) IV como en el DSM V (e incluso en sus versiones anteriores), se recolecta evidencia sobre las correlaciones altas entre la ausencia de empatía animal (que incluye el daño voluntario a fauna) y las tendencias psicopáticas. Ciertamente, no se está afirmando que este 
tipo de experimentos cría psicópatas, pero promueve una ausencia de empatía hacia los animales que puede derivar en una pérdida de empatía hacia otras personas.

Como si fuera poco, ya es sabido (Pérez, 2017; Asmat, Julca y Lucero, 2018; Echeverry, Cuartas y Henao, 2018) que si no hay motivación de aprendizaje, o incluso si la hay pero se da en una situación estresante o angustiante, el resultado final es nulo en términos de desempeño o eficacia. Esto configura no solo como arcaicas, caras y estresantes a las prácticas en cuestión, sino que, además, las perfila como totalmente ineficaces y contraproducentes para las personas que se pretende beneficiar.

De alguna manera, resulta increíble cómo autoridades o especialistas en educación no toman en consideración la interferencia cognitiva que podría producirse en un niño, quien durante su infancia probablemente ha tenido mascotas o contactos positivos con animales, al colocarlo en una situación de dañar o incluso matar a otro ser vivo similar a aquellos con los cuales ha tenido contacto. Y este enunciado no aplica exclusivamente a niños con mascotas, pues, como Bekoff y Peirce (2018) señalaron, los animales están presentes en nuestra sociedad bajo una enorme cantidad de formas, en libros, aplicaciones, películas, series, videos, publicidades, parques... y muchas veces los mensajes en los cuales aparecen son contradictorios para la infancia, dado que están al mismo nivel la publicidad de la venta de carne con la del alimento para tu mascota.

Por último, se refiere los beneficios de empelar prácticas alternativas en el uso de animales para la enseñanza de la biología, que no estarían marcadas por las desventajas señaladas anteriormente. Estas, como se verá, además de ser éticas son ecológicas, puesto que no atentan contra la biodiversidad local y promueven el desarrollo de la empatía por los animales, así como el cuidado del medio ambiente.

\section{Beneficios de una alternativa ecológica}

Para exponer los beneficios de una alternativa ecológica, se aludirán los tres puntos propuestos por Von Arcken (2017), a su vez apoyados en las concepciones de Magnusson et al. (2013) y Zapico (2019), de un uso limitado o reducido de los animales para la enseñanza de la biología. 
Ahora bien, esto no implica una tendencia directa a la desaparición de dicho uso, puesto que hay también gran documentación respecto a los efectos positivos que tanto en niños como adolescentes tiene la presencia de animales en su educación, en los ámbitos formal y no formal. Por ejemplo, Martín (2016) recopila una buena cantidad de estudios en los que se evidencia lo positivo de la terapia asistida con animales para estimular el interés y aprendizaje de infantes con trastorno del espectro autista. Por su parte, Tapia (2000) señala cómo los programas educativos que incluyen visitas presenciales a granjas, de forma recurrente, junto con un contacto fluido con distintos tipos de animales, mejoran no solo el interés y el desempeño académico de la niñez, sino también la dinámica del grupo y valores como la solidaridad, el interés por el otro, etc. Alarcón (2017), en el marco de las inteligencias múltiples, destaca, como potencial herramienta de aprendizaje y socialización, la incorporación (ocasional) de mascotas en el aula, en los contextos de materias como biología o ciencias naturales. También, Urbano (2018) enfoca la potencial implementación de un contacto más constante con animales y plantas en las aulas de educación primaria, como oportunidad para el desarrollo de una conciencia ecológica incipiente, educando para una empatía que abarque a todos los seres vivos.

Además, cada una de estas alternativas mencionadas no presenta los inconvenientes señalados en los apartados anteriores. Es decir, el conflicto no estaría en el empleo de animales como una manera de acceder al conocimiento, sino en el tipo de uso que se haga de ellos. Es precisamente el trato ético lo que perfila como superador a las propuestas que listamos en este apartado; tiene todos los beneficios pedagógicos, sin la necesidad de redundar en perjuicios para animales ni estudiantes.

\section{Conclusiones}

Se han analizado desde tres perspectivas los resultados de emplear animales para la enseñanza de la biología. Desde el punto de vista de la eficacia, se ha puesto en evidencia que la manipulación directa de fauna no obtiene mejores resultados (es más, alcanza peores) que las alternativas computarizadas. A su vez, en términos económicos, los clásicos laboratorios y sus insumos resultan extremadamente costosos cuando se los compara con los laboratorios web, donde no solo la ganancia es en términos económicos, sino también de aprendizaje. Por último, se puso énfasis en los aspectos psicológicos negativos que 
acarrea la manipulación directa de animales, los cuales van desde el estrés que redunda en falta de aprendizaje y motivación hasta la disminución de la empatía. Todos estos inconvenientes son evitables, al emplear modelos en los cuales el experimento pueda ser realizado de forma no directa ni invasiva en animales.

Dados estos tres argumentos, en el marco de un mundo donde tendemos cada vez más al individualismo y las problemáticas medioambientales obvias (la contaminación, el calentamiento global, la pérdida de la biodiversidad, entre otras) suelen ser ignoradas o tapadas por los medios y el ajetreo de las redes, la única posibilidad que tenemos de generar conciencia ecológica es a través de un cambio estructural en la forma de enseñar ciencias naturales y materias afines. Este posible cambio estará dado, en parte, por una educación que promueva la empatía hacia los animales desde los primeros años de la escolaridad. Tal nueva y viable perspectiva debe ser lo suficientemente amplia como para integrar a todos los actores: alumnos, docentes, padres, madres, la comunidad; a su vez, debe ser bastante transversal, para llegar desde las ciencias naturales hasta otras asignaturas.

Si se logra dimensionar que de la biodiversidad y su mantenimiento dependen el agua que tomamos, el aire que respiramos, así como la misma tierra sobre la cual construimos nuestras casas, quizá lleguemos a comprender como sociedad la importancia que puede tener algo que parece ser un detalle: el empleo de animales de formas no éticas en la educación de las futuras generaciones.

\section{Referencias}

Academia Nacional de Medicina. (1999). Guía para el cuidado y uso de animales de laboratorio. México: Editorial de la Academia Nacional de Medicina.

Acosta, R. B. (2019). La formación investigativa de los estudiantes a través de prácticas de laboratorio en el área de ciencia y tecnología. (Tesis de grado no publicada). Universidad Nacional Pedro Ruiz Gallo, Lambayaque, Perú.

Adell, J. (1996). Internet en educación: una gran oportunidad. Net Conexión, 11(5), 44-47.

Alarcón, A. (2013). Las inteligencias múltiples en el aula de Lengua Inglesa. (Tesis de maestría no publicada). Universidad de Almería, Almería, España. 
Asmat, P. E.; Dionicio, E. L. y Asmat, C. E. (2018). Programa de lenguaje gráfico-plástico para disminuir el estrés, en estudiantes de 5 años, Trujillo-2017. (Tesis de licenciatura no publicada). Universidad Católica de Trujulli Benedicto XVI, Trujillo, Perú. Recuperado de http://repositorio.uct.edu.pe/handle/123456789/421

Álvarez, S. M. y Carlino, P. C. (2004). La distancia que separa las concepciones didácticas de lo que se hace en clase: el caso de los trabajos de laboratorio en biología. Enseñanza de las ciencias: revista de investigación y experiencias didácticas, 22(2), 251-261.

Anderson, R. D. (2007). Teaching the theory of evolution in social, intellectual, and pedagogical context. Science Education, 91(4), 664-677.

Bustos, R. y Valenzuela, F. (2018). Evolución de aspectos bioéticos de la experimentación en animales: el origen del concepto "Cruelty free". Revista Chilena de Dermatología, 33(2), 48-51.

Calderón, S. E.; Núñez, P.; Di Laccio, J. L.; Iannelli, L. M. y Gil, S. (2015). Aulas-laboratorios de bajo costo, usando TIC. Revista Eureka sobre Enseñanza y Divulgación de las Ciencias, 12(1), 212-226.

Carin, A. A. y Sund, R. B. (1993). Teaching modern science. Nueva York: Merrill.

Campos, M. L.; Pasquali, C. y Peinado, S. (2008). Evaluación psicométrica de un instrumento de medición de actitudes proambientales en escolares venezolanos. Paradigma, 29(2), 1-9.

Candón-Mena, J. (2018). Riesgos y amenazas de Internet para la ciudadanía y la democracia. Más allá del alarmismo. CES contexto, $22,38-47$.

Castillo, J. F. G.; Leyva, J. C. G.; González, A. A. y Rosquett, L. S. B. (2005). Los costes de la investigación y la docencia cuando se utilizan animales de laboratorio. Revista Habanera de Ciencias Médicas, 4(5), 1-4.

Corredor, E. S. (2019). Unpacking "Gender Ideology" and the Global Right's Antigender Countermovement. Signs: Journal of Women in Culture and Society, 44(3), 613-638.

Coss, S. L.; Salvador, A. P. y Salazar, A. L. (2017). Calentamiento global, población, alimentación y sustentabilidad: límites en el contexto económico y social del sector agropecuario en México. 
Crecer Empresarial: Journal of Management and Development, $1,1-12$.

Cheverry, M. E.; Franco, J. y Henao, M. L. (2018). Factores psicosociales, autoeficacia percibida y engagement en los docentes de la Academia Nacional de Aprendizaje sedes Manizales y Pereira. Bogotá: Universidad de Manizales.

De Martínez, C. A. C. y De Osorio, A. M. (2015). Ética en investigación con animales: una actitud responsable y respetuosa del investigador con rigor y calidad científica. Revista Latinoamericana de Bioética, 8(15), 46-71.

De Pablo, M. (2016). La desinformación en la sociedad actual. Cuadernos.info, 3(1), 1-10.

Díaz, L. E.; Leyva, L. H.; Rodríguez, C. R. y Brito, L. M. (2012). Multimedia educativa para el perfeccionamiento del proceso enseñanza-aprendizaje de la asignatura Biología Celular. Revista Educación Médica del Centro, 4(1), 74-85.

Flichtentrei, D. (2017). Posverdad: la ciencia y sus demonios. IntraMed Journal, 6(1), 114-119.

Finkelstein, N. D.; Adams, W. K.; Keller, C. J.; Kohl, P. B.; Perkins, K. K.; Podolefsky, N. S. y LeMaster, R. (2005). When learning about the real world is better done virtually: A study of substituting computer simulations for laboratory equipment. Physical Review Special Topics-Physics Education Research, 1(1), 10-30.

Galaz, M. M. F.; Ayala, M. D. L. C. y Manrique, M. T. M. (2017). Estudio de validación del cuestionario de empatía emocional en niños. Psicumex, 7(2), 40-55.

Garzón, F. A. y Zárate, B. (2015). El aprendizaje de la bioética basado en problemas (ABBP): un nuevo enfoque pedagógico. Acta bioethica, 21(1), 19-28.

Gil, S. y Di Laccio, J. (2017). Smartphone una herramienta de laboratorio y aprendizaje: laboratorios de bajo costo para el aprendizaje de las ciencias. Latin-American Journal of Physics Education, 11(1), 5-14.

Herndon, J. M. (2015). Geoingeniería Tóxica en la Troposfera: evidencias de cenizas volantes de carbón-Consecuencias para la Salud Pública. Int. J. Environ. Res. Public Health, 12, 9375-9390.

Lane, R. (2016). Reading trans biology as a feminist sociologist. Transcender Studies Quarterly, 3(1-2), 185-191. 
Llach, C.; Del Mar, M. y Arís Giralt, A. (2005). Diseño de recursos multimedia de biología para un aprendizaje autónomo basado en problemas. Enseñanza de las ciencias, 3(1), 1-3.

Magnusson, W. et al. (2013). Biodiversidade e Monitoramento Ambiental Integrado/Biodiversity and Integrated Enviromental Monitoring. Manaus: Attem Editorial.

Maldonado-Villamizar, J. y Aquino-Guerra, A. (2016). Experimentación con biomodelos animales en ciencias de la salud. Avances en Biomedicina, 5(3), 173-177.

Maricela, L.; Restrepo, B.; Molina, C. y Fabiola, D. (2016). Curso experimental en biología y química empleando granjas rurales y recursos virtuales para la educación secundaria en la subregión del Nordeste Antioqueño. Medellín: Universidad Pontifica Bolivariana.

Martínez, N. (2015). Internet para propósitos educativos. Diálogos, 64(1), 31-45.

Millán, G. (2016). Víctimas de la educación. La ética y el uso de animales en la educación superior. Revista de la Educación Superior, 45(177), 177-182.

Mominó, J. M.; Sigalés, C. y Meneses, J. (2008). La escuela en la sociedad red. Internet en la educación primaria y secundaria. Barcelona: Ariel.

Muñoz-López, T.; Martínez-Sosa, V.; Cepeda-González, C. y Cervantes-Marmolejo, C. (2018). El Laboratorio Nómada del Agua, Con-Ciencia ambiental desde la Educación Básica The Nomadic Water Laboratory, Environmental Con-Science. Revista de Educación Básica, 2(6), 22-29.

Nosofsky, R. M. y McDaniel, M. A. (2019). Recommendations From Cognitive Psychology for Enhancing the Teaching of Natural-Science Categories. Policy Insights from the Behavioral and Brain Sciences, 6(1), 21-28.

Novo, M. (1996). La Educación Ambiental formal y no formal: dos sistemas complementarios. Iberoamericana de Educación, 11(1), $75-102$.

Ortíz, G. (2016). Víctimas de la educación. La ética y el uso de animales en la educación superior. Revista de la Educación Superior, 45(1), 147-170. 
Paredes, M. (2018). El aprendizaje activo, el aprendizaje basado en proyectos y la educación STEM. Cajica: Editorial del Departamento de Matemáticas.

Paterson, P. (2017). Calentamiento global y cambio climático en Sudamérica. Revista Política y Estrategia, 130, 153-188.

Pérez, A. M. (2017). Trastornos del Desarrollo y Dificultades del Aprendizaje. Alicante: Editorial de la Universidad de Alicante.

Pérez, A. y Florindo, R. (2003). Posibilidades y limitaciones de Internet como recurso educativo.Etic@net, 2, 1-12. Recuperado de https://www.ugr.es/ sevimeco/revistaeticanet/Numero2/Articulos/ Posibilidades $+y+$ limitaciones + de+Internet[1].pdf

Rigo, D. Y. y Donolo, D. S. (2013). Tres enfoques sobre inteligencia: un estudio con trabajadores manuales. Estudios de Psicología, 30(1), 39-48. Recuperado de http://dx.doi.org/10.1590/ S0103-166X2013000100005

Rodríguez-Marín, F.; Fernández-Arroyo, J.; Puig-Gutiérrez, M. y García-Díaz, J. E. (2017). Los huertos escolares ecológicos, un camino decrecentista hacia un mundo más justo. Enseñanza de las ciencias: revista de investigación y experiencias didácticas, 1 , 805-810.

Rutledge, M. L. y Mitchell, M. A. (2002). High school biology teachers' knowledge structure, acceptance \& teaching of evolution. The American Biology Teacher, 64(1), 21-28.

Sauvé, L. (1999). La educación ambiental entre la modernidad y la posmodernidad: en busca de un marco educativo de referencia integrador. Tópicos, 1(2), 7-27.

Ser-Martín, M. D. (2016). Beneficios de la Terapia Asistida con Animales en el Trastorno del Espectro Autista. (Tesis de grado no publicada). Universidad de Valladolid, Valladolid, España.

Sinatra, G. M.; Brem, S. K. y Evans, E. M. (2008). Changing minds? Implications of conceptual change for teaching and learning about biological evolution. Evolution: Education and outreach, l(2), 189-195.

Singer, P. (2018). Liberación animal: el clásico definitivo del movimiento animalista. Madrid: Taurus.

Tapia, M. N. (2000). Solidaridad como pedagogía. Buenos Aires: Ciudad Nueva. 
Universidad de Quilmes. (2017). Pliego de especificaciones técnicas. Recuperado de www.unq.edu.ar/advf/documentos/59f7162260907. docx

Urbano, N. B. (2018). La educación en valores a través de los seres vivos. Cartagena: Centro Universitario.

Vázquez, C. (2009). Equipación de un laboratorio escolar. Revista Innovación y Experiencia Educativa, 18, 1-10. Recuperado de https:// archivos.csif.es/archivos/andalucia/ensenanza/revistas/csicsif/ revista/pdf/Numero_18/CARLOS_VAZQUEZ_SALAS01.pdf

Vázquez-Cupeiro, S. (2015). Ciencia, estereotipos y género: una revisión de los marcos explicativos. Convergencia, 22(68), 177-202.

Novo, M. (2009). La educación ambiental, una genuina educación para el desarrollo sostenible. Revista de educación, 1, 195-217.

Viñao, A. (2000). Culturas escolares y reformas (sobre la naturaleza histórica de los sistemas e instituciones educativas). Revista Teias, 1(2), 1-25. Doi: 10.12957/teias

von Arcken, C. y Constanza, B. (2017). Algunos problemas relacionados con el uso de animales en docencia e investigación. Bogotá: Editorial Universidad de la Salle.

Zapico, M. G. (2019). Repensando el utilitarismo. 3. Estadística y filosofía de la opinión pública. El problema de las polémicas. Ensayos de Filosofía, 9(1), 1-11. 